\title{
Canine specific ELISA for coagulation factor VII
}

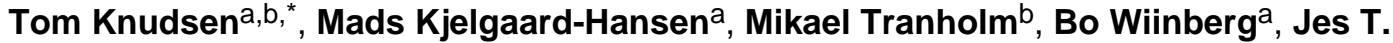 \\ Clausen $^{b}$, Jens Jacob Hansen ${ }^{b}$, Timothy C. Nichols ${ }^{c}$, Marianne Kjalke ${ }^{b}$, Asger L. Jensen ${ }^{d}$, \\ and Annemarie T. Kristensen ${ }^{\mathrm{a}}$ \\ aDepartment of Small Animal Clinical Sciences, Faculty of Life Sciences, University of \\ Copenhagen, Copenhagen DK-1870, Denmark
}

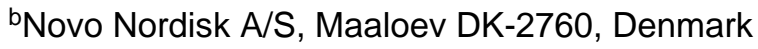

'Department of Pathology and Laboratory Medicine, University of North Carolina, Chapel Hill, NC 27516, USA

dDepartment of Basic Animal and Veterinary Sciences, Faculty of Life Sciences, University of Copenhagen, Frederiksberg C DK-1870, Denmark

\begin{abstract}
Canine coagulation factor VII (FVII) deficiency can be hereditary or acquired and may cause life threatening bleeding episodes if untreated. FVII procoagulant activity can be measured by FVII activity (FVII:C), but assays for measurement of canine specific FVII antigen (FVII:Ag) have not been available to date. In this study, a canine specific ELISA for measurement of FVII:Ag in plasma was developed and validated. The FVII:Ag ELISA correctly diagnosed homozygous and heterozygous hereditary FVII deficiency. Together with activity based assays, such as FVII:C, the FVII:Ag ELISA should be valuable in the diagnosis of hereditary canine FVII deficiency.
\end{abstract}

\section{Keywords}

FVII deficiency; Haemostasis; Coagulation factor; ELISA; Plasma; Immunoassays

\section{Introduction}

Canine coagulation factor VII (FVII) deficiency is a bleeding disorder with, primarily, a relatively mild phenotype (Mustard et al., 1962). Nevertheless, if untreated, it can result in life threatening bleeding and affected animals require intensive care (Spurling et al., 1974; Wheeler et al., 1984). This is also true in humans, where more than 100 unique genetic variants of hereditary FVII deficiency have been identified (Mariani et al., 2005). Clinical cases in humans range from asymptomatic to patients with severe haemorrhagic tendencies (Mariani et al., 1998) and significant mortality in neonates (Ragni et al., 1981).

Congenital FVII deficiency is well known in Beagles (Spurling et al., 1972), where the deficiency is caused by a G96E substitution in FVII (cFVII); as many as one third of Beagles may carry the defective gene (Wilhelm et al., 2001; Callan et al., 2006). Hereditary

\footnotetext{
C 2010 Elsevier Ltd. All rights reserved.

"Corresponding author. Tel.: +45 30751869; fax: +45 44663939. tmkn@ novonordisk.com (T. Knudsen).

Conflict of interest statement

Tom Knudsen, Mikael Tranholm, Jes T. Clausen, Jens Jacob Hansen and Marianne Kjalke are employees of Novo Nordisk A/S, which provided part funding for the study. The authors state that they have no financial or intellectual property right interests that may be perceived as conflicting in regard to the work reported in the present manuscript.
} 
FVII deficiency (G96E variant) is also seen in other breeds, such as the Alaskan Klee Kai (Kaae et al., 2007). FVII deficiency may also be acquired, either due to decreased production of functional FVII (e.g. rodenticide poisoning and hepatic diseases), due to consumption (e.g. disseminated intravascular coagulation, DIC) or due to dilution coagulopathies secondary to fluid resuscitation. One case of combined congenital and acquired FVII deficiency has been reported as a result of rodenticide poisoning in a Beagle (Daly and Giger, 2007).

Quantification of canine FVII clotting activity in prothrombin time (PT)-based assays has proven feasible but difficult (Mischke et al., 2003). To detect canine FVII deficiency by means of the standard PT assay, the functional factor level must be <39\% (Mischke et al., 2003), i.e. decreased by at least $18 \%$ below the normal reference (Mischke, 2001). Due to a lack of appropriate assays, precise canine FVII:Ag levels have not been determined (Callan et al., 2006). This lack of sufficiently sensitive assays is relevant both to canine medicine and experimental models used in preclinical research (Callan et al., 2006). Therefore, an accurate assay to detect FVII antigen (FVII:Ag) in dogs is required.

The aims of the current study were to develop and validate a canine specific ELISA to accurately measure FVII:Ag in canine plasma, to apply the FVII:Ag assay in the diagnostic work up of canine hereditary FVII deficiency and to determine whether the FVII:Ag assay is superior to existing PT and FVII activity (FVII:C) assays in accurately identifying carriers of hereditary FVII deficiency.

\section{Materials and methods}

\section{Animals}

Plasma samples from 87 dogs were included in the study. Of these 87 dogs, 71 were client owned dogs submitted to the Onderstepoort Veterinary Academic Hospital, Faculty of Veterinary Science, University of Pretoria, and 16 were colony dogs from two different colonies: four FVII deficient homozygotes from the Chapel Hill colony at the University of North Carolina and 12 obtained from a commercial supplier for other purposes (3 FVII deficient heterozygotes and 9 unaffected). The colony dogs were housed in the Chapel Hill colony (FVII deficient homozygotes) or in an enriched environment at Novo Nordisk, Denmark (heterozygotes and unaffected dogs). Colony dogs were genotyped according to Callan et al. (2006).

A plasma pool from eight clinically healthy client owned dogs ( 3 mixed breed, 2 Border Terriers, 1 Labrador mix, 1 Whippet, 1 Shetland Sheepdog) was used as FVII:Ag reference material and for calibration of the FVII:C assay. The plasma pool was produced at the Department of Small Animal Clinical Sciences, Faculty of Life Sciences, University of Copenhagen. The dogs were characterised 'free from overt disease' based on clinical examination, complete blood count $(\mathrm{CBC})$, biochemistry and a coagulation profile consisting of prothrombin time (PT), activated partial thromboplastin time (aPTT), DDimer, fibrinogen and platelet count.

Four mice (RBF/DnJ, Jackson Laboratory), housed at Novo Nordisk, Denmark, were used for antibody production. Experiments were approved by the Animal Use and Care Committee at the University of Pretoria (approval number V010/07), the Institutional Animal Care and Use Committee at the University of North Carolina (approval number 08-134.0-B), the Danish Animal Experiments Council and the Danish Ministry of Justice (approval numbers: dogs: 2007/561-1276; mice: 2007/561-1413) and by the Animal Use and Ethics committee of the Department of Small Animal Clinical Sciences, Faculty of Life 
Sciences, University of Copenhagen. Written consent was obtained before sampling of client owned dogs.

\section{Sample collection}

Whole blood was collected into citrated plastic tubes (Vacutainer, Greiner Bio-One) by careful venipuncture using minimum stasis and a $21 \mathrm{G}$ butterfly needle. Plasma was isolated by centrifugation, separated into aliquots, frozen immediately after sampling and stored at $-80^{\circ} \mathrm{C}$ until it was shipped on dry ice to the site of analysis. After arrival in Denmark, plasma samples were stored at $-80^{\circ} \mathrm{C}$ until the time of the assay.

\section{Canine specific monoclonal anti-FVII antibodies}

Canine specific monoclonal antibodies (mAbs) were raised in mice by immunisation with recombinant canine FVIIa (rcFVIIa) (Knudsen et al., 2010) using standard procedures (Appendix A supplementary material).

\section{ELISA development}

Microtitre plates (96-wells/plate) were coated with the capture antibody ('F11') at $0.5 \mu \mathrm{g}$ / $\mathrm{mL}$ (antibody concentration initially optimised in a matrix setup) in $100 \mu \mathrm{L}$ coating buffer $\left(10 \mathrm{mM} \mathrm{NaHCO}_{3}, \mathrm{pH}\right.$ 9.6) overnight at $4{ }^{\circ} \mathrm{C}$. Blocking was completed by incubation for 15 min at room temperature (RT) with $300 \mu \mathrm{L}$ washing buffer containing $20 \mathrm{mM} 4-(2-$ hydroxyethyl)-1-piperazineethanesulphonic acid (HEPES), $150 \mathrm{mM} \mathrm{NaCl}$ and $0.02 \%$ Tween $80(\mathrm{pH}$ 7.4), before washing the plates three times with $300 \mu \mathrm{L}$ washing buffer using an automated plate washer (ELx50, BioTek Instruments).

Samples were pre-diluted 1:100 with dilution buffer consisting of washing buffer with $0.1 \%$ W/V bovine serum albumin (BSA) substituted for Tween 80. Pre-dilution was optimised in initial experiments; although 1:100 was selected as the optimal pre-dilution, alternative predilutions, e.g. 1:25 of FVII deficient plasma, also performed well in the assay. Two hundred microlitres of pre-diluted samples were incubated for $60 \mathrm{~min}$ at RT in each test well of the microtitre plate. After washing the plate three times using $300 \mu \mathrm{L}$ washing buffer each time, $200 \mu \mathrm{L}$ of $1.0 \mu \mathrm{g} / \mathrm{mL}$ horse radish peroxidase (HRP)-conjugated detector antibody ('F20HRP'; HRP conjugated using Lightning-Link HRP, Innova Biosciences) was added to the wells and incubated for $30 \mathrm{~min}$ at RT; the concentration of the detector antibody was optimised using a matrix setup. The plate was washed three times again, then $100 \mu \mathrm{L}$ of a commercial 3,3',5,5'-tetramethylbenzidine (TMB) solution (T4444, Sigma-Aldrich) was added to wells and incubated for $20 \mathrm{~min}$ at RT. The enzymatic reaction was quenched by addition of $100 \mu \mathrm{L} 1 \mathrm{M}$ sulphuric acid.

Colour development was quantified using a microplate spectrophotometer (SpectraMax 340, Molecular Devices), detecting the optical density at $450 \mathrm{~nm}\left(\mathrm{OD}_{450 \mathrm{~nm}}\right)$. Sample FVII:Ag was calculated using SOFTmax Pro (Molecular Devices) by comparing $\mathrm{OD}_{450 \mathrm{~nm}}$ signals from a seven point second order standard curve created with $0.3-30 \mathrm{nmol} / \mathrm{L}$ recombinant canine FVII. The stock concentration of the reference standard was determined using a micro-volume spectrophotometer (Knudsen et al., 2010).

\section{Analytical performance}

Inaccuracy-Inaccuracy was assessed in spike-recovery and dilution linearity experiments. In spike-recovery experiments, zymogen recombinant canine FVII (rcFVII) was spiked into pooled FVII deficient plasma from the four G96E homozygotes included in this study, in human normal plasma (HemosIL Calibration plasma, Instrumentation Laboratory), human FVII deficient plasma (Helena Laboratories), in dilution buffer and in 
Tris-buffered saline (TBS)/BSA (50 mM Tris, $100 \mathrm{mM} \mathrm{NaCl,} 1 \%$ V/V BSA, pH 7.4) to create samples with low $(3 \mathrm{nM})$, medium $(15 \mathrm{nM})$ and high $(27 \mathrm{nM})$ concentrations of FVII:Ag.

The samples were then assayed in duplicate in three separate runs and the baseline corrected recovery was reported as a percentage relative to the theoretical FVII:Ag content of the sample. Dilution linearity was assessed in three separate runs with duplicate measurements of pooled normal canine plasma serially diluted to yield samples with $100 \%$ (undiluted), $75 \%, 50 \%, 25 \%, 10 \%, 5 \%$ and $1 \%$ FVII:Ag.

Detection limit and analytical imprecision-The detection limit was defined as the mean signal plus three standard deviations (SD) obtained from a buffer blank assayed in 10 replicates. To survey analytical imprecision in terms of the intra- and inter-assay coefficient of variation $(\mathrm{CV})$, three specimens with low, medium and high (i.e. normal) concentrations of FVII:Ag, prepared by mixing plasma from unaffected wild type (wt/wt) and FVII deficient (G96E/G96E) dogs, were analysed in 10 replicates for intra-assay characterisation and in duplicates in five individual experiments for inter-assay characterisation.

Freeze-thaw and stability at $-\mathbf{2 0}^{\circ} \mathbf{C}-$ Freeze-thaw stability was examined by submitting samples with different plasma FVII:Ag content to repeated freezing on dry ice for approximately $3 \mathrm{~min}$ then thawing in a water bath at room temperature, before assaying all samples in duplicates in the same run. Aliquots were prepared from the same starting material and then one sample was taken aside (before freeze-thawing) and kept at $4{ }^{\circ} \mathrm{C}$. The remaining samples were then submitted to the first freeze-thaw cycle. Then, one additional sample was taken aside and kept at $4{ }^{\circ} \mathrm{C}$, together with the non-frozen sample. The procedure was repeated until all samples had undergone $0-5$ freeze-thaw cycles. Total preanalytical processing time was approximately $30 \mathrm{~min}$. The experiment also included an identical setup with rcFVII at 30,15 and $7.5 \mathrm{nmol} / \mathrm{L}$ in TBS/BSA buffer. Stability at $-20{ }^{\circ} \mathrm{C}$ was evaluated by repeated duplicate measurements, 125 days apart, of pooled normal canine plasma kept at $-20^{\circ} \mathrm{C}$.

Measurements in the presence of human FVIla-The ability of the assay to correctly measure canine FVII:Ag in the presence of recombinant human FVIIa (rhFVIIa) was assessed in experiments $(n=3)$ with spiking of normal canine plasma with rhFVIIa at $25,50,100$ and $250 \mathrm{nmol} / \mathrm{L}$ (duplicate measurements for each concentration in each experiment).

Conventional coagulation profile and FVII:C assay-All haemostasis tests were performed at the Central Laboratory, Department of Small Animal Clinical Sciences, Faculty of Life Sciences, University of Copenhagen, employing commercial reagents in a validated setup with an automated coagulometer (ACLTop 500, Instrumentation Laboratory) (Wiinberg et al., 2009): aPTT (SynthAFax, Instrumentation Laboratory), PT (RecombiPlasTin 2G, Instrumentation Laboratory), D-dimer (D-Dimer Single Tests, NycoCard READER II, Medinor A/S), fibrinogen (RecombiPlasTin 2G, Instrumentation Laboratory) and FVII activity (FVII:C). FVII:C measurements were completed (in a PT based assay) with a commercially available human test system comprising human tissue factor (Recombi-PlasTin 2G, Instrumentation Laboratory) and human FVII depleted substrate plasma (Factor VII deficient plasma, Instrumentation Laboratory) and calibrated with a canine plasma pool.

Method comparison: FVII:C vs. FVII:Ag in samples from dogs with varying FVII levels-As part of the evaluation of analytical performance, the ability of the FVII:Ag 
assay to measure canine FVII was assessed indirectly by performing a method comparison of parallel FVII:C and FVII:Ag measurements where high correlation was expected, as previously demonstrated with human samples (Patrassi et al., 1993; Takamiya and Yoshioka, 1996; Hunault et al., 1997). Seventy-one samples from dogs with clinical babesiosis available from another study were measured in both assays to challenge the FVII:Ag assay with samples expected to vary widely in FVII content. To allow direct comparison of FVII:C (activity) and FVII:Ag (antigen), results were given relative to a normal pool (\%). Method comparison was performed according to Jensen and KjelgaardHansen (2006).

\section{Comparison of the diagnostic performance of prothrombin time, FVII:C and FVII:Ag assays in detection of hereditary FVII deficiency-To assess which assay} potentially would be best suited to diagnose hereditary FVII deficiency (G96E variant), plasma from the 16 colony dogs (4 G96E/G96E homozygotes, 3 G96E/wt heterozygotes, 9 wt/wt homozygotes) were assayed for PT, FVII:C and FVII:Ag.

Acceptance criteria and statistical analyses-Analytical imprecision was assessed by standard methods (Jensen and Kjelgaard-Hansen, 2010). The intra-assay and inter-assay inaccuracies measured as CV were deemed to be acceptable when $<10 \%$ and $<20 \%$, respectively.

A detection limit of 5\% FVII:Ag (compared to a normal pool) was defined as acceptable, since affected Beagles with hereditary FVII deficiency have been reported to have plasma FVII:C $\leq 5 \%$ normal (Callan et al., 2006; Withnall and Giger, 2006). Thus, FVII:Ag $\leq 5 \%$ would imply an unequivocal diagnosis of FVII deficiency.

Accuracy was deemed to be acceptable if recovery was $90-110 \%$ in spike-recovery experiments and if data from dilution linearity experiments could be fitted by linear regression with a correlation coefficient $\left(r^{2}\right)$ above $>0.99$ to a straight line not significantly different from a line representing agreement (FVII: $\left.\mathrm{Ag}_{[\text {theoretical] }}=\mathrm{FVII}: \mathrm{Ag}_{[\text {measured] }}\right)$.

Freeze thaw stability and storage stability at $-20{ }^{\circ} \mathrm{C}$ were judged to be acceptable if mean recorded FVII:Ag values of thawed and stored samples were within $90-110 \%$ of mean FVII:Ag from the unfrozen original sample, respectively.

Agreement between FVII:C and FVII:Ag measurements was assessed by Passing- Bablock linear regression and was deemed to be acceptable when the slope and intercept were not statistically different from 1 and 0 , respectively. Cusum test was used to identify potential deviation from linearity. The nature of possible disagreement was assessed by BlandAltman plotting with criteria for combined inherent imprecision (Jensen and KjelgaardHansen, 2006).

To evaluate the discriminatory power of PT, FVII:C and FVII:Ag assays in distinguishing between wt homozygotes (wt/wt), FVII deficient heterozygotes (G96E/wt) and FVII deficient homozygotes (G96E/G96E), the measured levels of PT, FVII:C and FVII:Ag in the three groups were compared by means of the Mann-Whitney $U$-test and receiver operator characteristic (ROC) curve analysis (Gardner and Greiner, 2006). The acceptance limit for diagnostic capacity was set at $\mathrm{AUC}_{\mathrm{ROC}}>0.5$ and classified as good with an $\mathrm{AUC}_{\mathrm{ROC}} \searrow 0.9$. Tests with $A \mathrm{AU}_{\mathrm{ROC}} \searrow 0.5$ were compared to detect possible superiority of one test vs. another, i.e. test $\mathrm{A}$ is superior to test $\mathrm{B}$ if $\mathrm{AUC}_{\mathrm{ROC}(\text { test } \mathrm{A})}>\mathrm{AUC}_{\mathrm{ROC}}$ (test $\mathrm{B}$ ).

All data were reported as 'mean' or 'mean with $95 \%$ confidence interval $\left(\mathrm{CI}_{95}\right)^{\prime}$ unless otherwise noted. Where single experiments were reported, 'means' represents the mean of 
duplicate measurements. Similarly, when experiments were repeated, reported 'means' represent means of mean duplicate values from all experiments. Statistical significance was set at $P<0.05$. Calculations were performed in GraphPad Prism 5.02, Medcalc 6.0 or Microsoft Office Excel 2003 SP3.

\section{Results}

\section{Analytical performance}

A minimally activated rcFVII preparation (Knudsen et al., 2010) was used for standard curves (Fig. 1); control experiments replacing zymogen rcFVII with activated rcFVIIa did not identify different binding of rcFVII and rcFVIIa by the two mAbs used in the ELISA (data not shown). Thus, the ELISA measured canine FVII:Ag as total FVII:Ag + FVIIa:Ag. The detection limit, using a 1:100 sample pre-dilution, was 1.2\% FVII:Ag compared to normal pooled plasma (FVII:Ag (Blanks) $_{0} 0.918 \pm 0.093 \%$; mean $\pm \mathrm{SD}$ ). The assay could be modified with better resolution at lower FVII:Ag concentrations by applying less dilute sample formulations, e.g. by using a 1:25 pre-dilution instead of the 1:100 used in the present study, without adverse effects on precision and reproducibility (data not shown). Analytical imprecision within and between assays was acceptable for all samples (Table 1). Accuracy criteria were held for samples with low, medium and high FVII:Ag in spikerecovery experiments; mean recovery was $97-106 \%$ in canine FVII deficient plasma, 91$105 \%$ in human plasma and $96-102 \%$ in buffer (Table 2).

Dilution linearity met criteria; $\mathrm{CI}_{95}$ for slope and intercept were $[0.956 ; 1.024]$ and $[-0.275$; 0.518], respectively (Fig. 2). Freeze-thaw stability was acceptable for all samples submitted to $\mathcal{2}$ freeze-thaw cycles. Specimens with low FVII:Ag did not hold stability criteria with three or more freeze-thaw cycles. Specimens with medium and high FVII:Ag held criteria through all five freeze-thaw cycles (Table 3). Freezer stability at $-20^{\circ} \mathrm{C}$ was acceptable throughout the 4 month study period. Measurements of FVII:Ag in normal plasma spiked with $25-250 \mathrm{nmol} / \mathrm{L}$ rhFVIIa ranged from 98 to $103 \%$ of unspiked controls, demonstrating no cross reactivity of the applied antibodies to rhFVIIa.

\section{Method comparison: FVII:C vs. FVII:Ag in samples from dogs with varying FVII levels}

Activity and antigen based measurements of FVII, i.e. FVII:C and FVII:Ag, respectively, in 71 clinical samples from dogs with clinical babesiosis (hence at risk of having acquired FVII deficiency), spanned a range from 6.7\% FVII:C and 9.1\% FVII:Ag to $\geq 150 \%$ FVII:C (upper limit of working range) and FVII:Ag when compared to a normal pooled plasma with a FVII concentration of $19.4 \mathrm{nmol} / \mathrm{L}$ FVII:Ag. Measurements were significantly correlated between the two methods; Passing-Bablock regression yielded $\mathrm{CI}_{95}[0.97 ; 1.34]$ for slope and [-17.81; 5.84] for intercept (Fig. 3A). No systematic error was identified; linear regression of residuals yielded $\mathrm{CI}_{95}[-0.16 ; 0.16]$ for slope and $[-12.73 ; 12.73]$ for intercept. Although no systematic error was identified, a total of 43 residuals (61\%) were outside, $19(27 \%)$ above and 24 (34\%) below the bounds of the expected interval defined by the combined inherent imprecision of the FVII:C and FVII:Ag assays (Fig. 3B), demonstrating random disagreement in excess of combined imprecision.

\section{Comparison of the diagnostic performance of PT, FVII:C and FVII:Ag assays for detection of hereditary FVII deficiency}

With FVII:C and FVII:Ag, but not PT, significantly different mean FVII levels were identified between wt/wt homozygotes and wt/G96E heterozygotes, i.e. between groups of wild type and carrier dogs. At the individual dog level, FVII:C values overlapped between wild type and carrier animals, while this was not observed for FVII:Ag. In comparison, 
different mean PT, FVII:C and FVII:Ag values were identified between wt/wt (healthy wild type) and G96E/G96E (affected FVII deficient) homozygotes (Table 4 and Fig. 4A-C).

Using ROC analysis, both FVII:C and FVII:Ag assays had significant diagnostic abilities in distinguishing between healthy wild type homozygotes (wt/wt) and heterozygote carriers of the FVII deficiency gene (wt/G96E), with both FVII:C and FVII:Ag demonstrating high diagnostic performance: $\mathrm{AUC}_{\mathrm{ROC}(\mathrm{FVII}: \mathrm{C})}=0.936$ and $\mathrm{AUC}_{\mathrm{ROC}(\mathrm{FVII}: \mathrm{Ag})}=1.0$ (Fig. $4 \mathrm{E}$ and F). No significant difference was demonstrated between diagnostic performances of FVII:C and FVII: $\mathrm{Ag}\left(\triangle \mathrm{AUC}_{\mathrm{ROC}}=[-0.070 ; 0.144], P=0.5\right)$, i.e. the null hypothesis $\mathrm{H}_{0}$ : $\mathrm{AUC}_{\mathrm{ROC}(\mathrm{FVII}: \mathrm{C})}=\mathrm{AUC}_{\mathrm{ROC}(\mathrm{FVII}: \mathrm{Ag})}$ was not rejected.

In contrast to FVII:C and FVII:Ag, PT was of no appreciable value in distinguishing between dogs as either healthy wild type or carriers: $\mathrm{AUC}_{\mathrm{ROC}}=0.741\left(\mathrm{CI}_{95}:[0.453 ; 1.0] ; P\right.$ $=0.230)($ Fig. 4D). When used to distinguish between healthy wild type (wt/wt) and FVII deficient homozygotes (G96E/G96E), all three assays demonstrated diagnostic abilities as demonstrated by an $\mathrm{AUC}_{\mathrm{ROC}}$ of $1.0(P<0.01)$ for PT, FVII:C and FVII:Ag, respectively.

\section{Discussion}

Timely diagnosis of hereditary FVII deficiency is important for optimal treatment of individual animals and tools are needed to guide rational breeding, with the aim of limiting or eliminating hereditary FVII deficiency in dog populations (Callan et al., 2006). To date, diagnosis of hereditary FVII deficiency has been based on gene analysis detecting a single causative mutation in exon 5 of the FVII gene ( $F 7$ ), because measurements of plasma FVII coagulant activity have not been able to unambiguously detect carrier dogs and a canine FVII ELISA has not been available (Callan et al., 2006). The validated FVII:Ag assay reported here offers an alternative and precise method to quantify canine FVII in dog plasma.

The ELISA developed in this study used a matched pair of murine mAbs to canine FVII and measured canine FVII:Ag as total FVII + FVIIa antigen. Imprecision, in terms of intra- and inter-assay variation was acceptable for plasma samples with low, medium and high FVII:Ag. To enhance clinical applicability, a reference population must be sampled before a normal FVII:Ag reference range can be established (Geffre et al., 2009). However, from the present study, it seems that normal canine FVII:Ag may be approximately $20 \mathrm{nmol} / \mathrm{L}$, which is twice the concentration in humans (Fair, 1983). The antibodies used were counterscreened against rhFVIIa and experiments showed that FVII:Ag measurements were unaffected by $250 \mathrm{nmol} / \mathrm{L}$ rhFVIIa. Thus, the assay will correctly measure canine FVII, even in dogs that have been treated with rhFVIIa (Withnall and Giger, 2006).

Additionally, no matrix effect was observed in spike-recovery experiments and hence the assay should reliably measure FVII:Ag in a wide range of sample materials. Good freezethaw and stability at $-20{ }^{\circ} \mathrm{C}$ was observed; accordingly, in a clinical study, it should be feasible to collect samples over a 4 month period and to analyse them in batches after completing the sample collection phase.

To demonstrate the ability to measure FVII:Ag over a wide range of concentrations, the study included a comparison of the FVII:C and FVII:Ag assays as part of the validation procedure. In both assays, plasma FVII was measured in samples from dogs with clinical babesiosis expected to have varying levels of plasma FVII due to consumption and haemoconcentration. As expected from human studies, FVII:C and FVII:Ag were correlated and there was no systematic error. However, the random difference between plasma FVII as assessed by FVII:C and FVII:Ag was too large to be explained by the combined inherent 
imprecision of the assays, i.e. the source of random variation beyond criteria was not directly related to assay imprecision.

To assess the diagnostic performance of PT, FVII:C and FVII:Ag for detection of hereditary FVII deficiency, samples from 16 dogs were run in parallel in all three assays. For an assay to be applicable in a situation where breeding is directed by its results, the assay must be both very sensitive and very specific. ROC curve analysis demonstrated high discriminative performance of both FVII:C and FVII:Ag, which were significantly superior to standard PT measurements in detecting heterozygous carriers of G96E compared to wt/wt homozygotes. Overlap between measurements from wt/wt homozygotes and wt/G96E heterozygotes was seen with FVII:C, but not FVII:Ag; however, it was not possible to demonstrate a superior performance of FVII:Ag over FVII:C, or vice versa, statistically due to the small sample size. However, had a previously reported reference range for normal canine FVII:C, i.e. 57$181 \%$ (Mischke, 2001), been applied in this study, all three wt/G96E heterozygotes would have been falsely classified as normal.

A purely qualitative FVII deficiency variant with normal FVII:Ag levels, e.g. a canine analogue to human FVII A294V (Toso et al., 2002), would potentially go undetected by the ELISA but would be identified by specific genetic testing and in activity assays.

Accordingly, activity based assays, such as FVII:C, should not be omitted in the diagnostic work up of canine FVII deficiency. Rather, FVII:C and FVII:Ag measurements should be viewed as complementary in detecting FVII deficiency and genetic testing is still valuable for confirming diagnosis in hereditary disease. Furthermore, the ELISA is not expected to detect all cases of FVII deficiency acquired as a consequence of vitamin-K antagonism, since FVII:Ag levels can be near normal (Takamiya and Yoshioka, 1996) and the antibodies applied were raised against rcFVIIa lacking the Gla-domain. To enable specific detection of vitamin-K antagonism, resulting in aberrant post-translational Gla-domain processing, a $\mathrm{Ca}^{2+}$ dependent FVII antibody could be developed (Nakagaki et al., 1991) and applied in the ELISA.

Taken together, FVII:Ag and also FVII:C, as configured in the present study, appears to be valuable in the initial diagnostic work up of hereditary canine FVII deficiency, especially in situations where time to diagnosis is essential or where the genetic test, targeting a single mutation will fail, e.g. in promoting the discovery of new causative mutations. Activity, antigen and gene directed assays will be valuable in complementing each other when applied rationally in a panel to identify canine FVII deficiency.

\section{Conclusions}

A canine specific FVII ELISA was developed and validated to accurately measure FVII:Ag in plasma. The FVII:Ag assay correctly diagnosed both homozygous and heterozygous hereditary FVII deficiency and, together with FVII:C and gene analyses, should be valuable in the diagnostic work up of hereditary canine FVII deficiency. FVII:Ag and FVII:C compared favourably with PT when the diagnostic performance of these three assays to detect heterozygous hereditary FVII deficiency was compared directly. A larger prospective study, with establishment of absolute reference intervals, is needed to compare the diagnostic performance of FVII:Ag and FVII:C to detect heterozygous hereditary FVII deficiency against the current gold standard PCR method.

\section{Supplementary Material}

Refer to Web version on PubMed Central for supplementary material. 


\section{Acknowledgments}

The authors wish to thank Drs. Amelia Goddard, Liza Koster and Johan Schoeman, University of Pretoria, South Africa, for providing samples from dogs with clinical babesiosis, Helle Tirpan (Novo Nordisk A/S) and Anette Urbrand Martinsen (Central Laboratory, Faculty of Life Sciences, University of Copenhagen) for expert technical assistance. The authors also wish to thank the participating staff in the Department of Small Animal Clinical Sciences, Faculty of Life Sciences, University of Copenhagen, Denmark, the Francis Owen Blood Research Laboratory, University of North Carolina, Chapel Hill, USA, and Novo Nordisk A/S, Denmark. The colony of dogs at UNC was supported by NIH/NHLBI Grant R24HL063098-12. The study was financially supported through an unrestricted grant to Tom Knudsen by The Faculty of Life Sciences, University of Copenhagen, The Danish Ministry of Science, Technology and Innovation and Novo Nordisk A/S, Denmark.

The authors are investigating the possibility of making the two monoclonal canine specific FVII antibodies reported herein commercially available. In the meanwhile we will provide these antibodies for research and diagnostic purposes under a material transfer agreement. Please address requests to the corresponding author.

\section{References}

Callan MB, Aljamali MN, Margaritis P, Griot-Wenk ME, Pollak ES, Werner P, Giger U, High KA. A novel missense mutation responsible for factor VII deficiency in research Beagle colonies. Journal of Thrombosis and Haemostasis. 2006; 4:2616-2622. [PubMed: 16961583]

Daly ML, Giger U. A rodenticide exposed and bleeding Beagle dog with hereditary factor VII deficiency. Journal of Veterinary Emergency and Critical Care. 2007; 17:170-174.

Fair DS. Quantitation of factor VII in the plasma of normal and warfarin-treated individuals by radioimmunoassay. Blood. 1983; 62:784-791. [PubMed: 6882924]

Gardner IA, Greiner M. Receiver-operating characteristic curves and likelihood ratios: improvements over traditional methods for the evaluation and application of veterinary clinical pathology tests. Veterinary Clinical Pathology. 2006; 35:8-17. [PubMed: 16511785]

Geffre A, Friedrichs K, Harr K, Concordet D, Trumel C, Braun JP. Reference values: a review. Veterinary Clinical Pathology. 2009; 38:288-298. [PubMed: 19737162]

Hunault M, Arbini AA, Lopaciuk S, Carew JA, Bauer KA. The Arg353Gln polymorphism reduces the level of coagulation factor VII. In vivo and in vitro studies. Arteriosclerosis, Thrombosis, and Vascular Biology. 1997; 17:2825-2829.

Jensen AL, Kjelgaard-Hansen M. Method comparison in the clinical laboratory. Veterinary Clinical Pathology. 2006; 35:276-286. [PubMed: 16967409]

Jensen, AL.; Kjelgaard-Hansen, M. Diagnostic test validation. In: Weiss, DJ.; Wardrop, KJ., editors. Schalm's Veterinary Hematology. 6. Blackwell; 2010. p. 1027-1033.

Kaae JA, Callan MB, Brooks MB. Hereditary factor VII deficiency in the Alaskan Klee Kai dog. Journal of Veterinary Internal Medicine. 2007; 21:976-981. [PubMed: 17939552]

Knudsen T, Kristensen AT, Sorensen BB, Olsen OH, Stennicke HR, Petersen LC. Characterization of canine coagulation factor VII and its complex formation with tissue factor: canine-human crossspecies compatibility. Journal of Thrombosis and Haemostasis. 2010; 8:1763-1772. [PubMed: 20524980]

Mariani G, Herrmann FH, Dolce A, Batorova A, Etro D, Peyvandi F, Wulff K, Schved JF, Auerswald G, Ingerslev J, Bernardi F. Clinical phenotypes and factor VII genotype in congenital factor VII deficiency. Thrombosis and Haemostasis. 2005; 93:481-487. [PubMed: 15735798]

Mariani G, Lo CL, Bernardi F, Pinotti M. Molecular and clinical aspects of factor VII deficiency. Blood Coagulation and Fibrinolysis. 1998; 9:S83-S88. [PubMed: 9819034]

Mischke R. Optimization of coagulometric tests that incorporate human plasma for determination of coagulation factor activities in canine plasma. American Journal of Veterinary Research. 2001; 62:625-629. [PubMed: 11327476]

Mischke R, Diedrich M, Nolte I. Sensitivity of different prothrombin time assays to factor VII deficiency in canine plasma. The Veterinary Journal. 2003; 166:79-85. [PubMed: 12788020]

Mustard JF, Secord D, Hoeksema TD, Downie HG, Rowsell HC. Canine factor-VII deficiency. British Journal of Haematology. 1962; 8:43-47. [PubMed: 14477610] 
Nakagaki T, Foster DC, Berkner KL, Kisiel W. Initiation of the extrinsic pathway of blood coagulation: evidence for the tissue factor dependent autoactivation of human coagulation factor VII. Biochemistry. 1991; 30:10819-10824. [PubMed: 1932002]

Patrassi GM, Sartori MT, Viero M, Boeri G, Simioni P, Bassi N, Piccinni P, Girolami A. Protein C, factor VII and prothrombin time as early markers of liver function recovery or failure after liver transplantation. Blood Coagulation and Fibrinolysis. 1993; 4:863-867. [PubMed: 8148479]

Ragni MV, Lewis JH, Spero JA, Hasiba U. Factor VII deficiency. American Journal of Hematology. 1981; 10:79-88. [PubMed: 7258184]

Spurling NW, Burton LK, Peacock R, Pilling T. Hereditary factor-VII deficiency in the beagle. British Journal of Haematology. 1972; 23:59-67. [PubMed: 5045961]

Spurling NW, Peacock R, Pilling T. The clinical aspects of canine factor-VII deficiency including some case histories. Journal of Small Animal Practice. 1974; 15:229-239. [PubMed: 4449220]

Takamiya O, Yoshioka A. Factor VII binding to tissue factor in plasma from warfarin-treated individuals. Thrombosis Research. 1996; 81:657-663. [PubMed: 8868516]

Toso R, Pinotti M, High KA, Pollak ES, Bernardi F. A frequent human coagulation factor VII mutation (A294V, c152) in loop 140s affects the interaction with activators, tissue factor and substrates. Biochemical Journal. 2002; 363:411-416. [PubMed: 11931672]

Wheeler SL, Weingand KW, Thrall MA, Berg RJ, Schwarz PD, Olson PN. Persistent uterine and vaginal hemorrhage in a beagle with factor VII deficiency. Journal of the American Veterinary Medical Association. 1984; 185:447-448. [PubMed: 6469849]

Wiinberg B, Jensen AL, Rozanski E, Johansson PI, Kjelgaard-Hansen M, Tranholm M, Kristensen AT. Tissue factor activated thromboelastography correlates to clinical signs of bleeding in dogs. The Veterinary Journal. 2009; 179:121-129. [PubMed: 17920966]

Wilhelm, C.; Czwalinna, A.; Wermes, C.; Bartzkowiak, N.; Mischke, R.; Ganser, A.; von Depka, M. Isolation and characterization of the canine factor VII gene of the Beagle dog with factor VII deficiency. Thrombosis and Haemostasis Suppl; Proceedings of the XVIII Annual Congress of the International Society on Thrombosis and Haemostasis; Paris, France. July 2001; 2001. p. 577(Abstract)

Withnall E, Giger U. Effects of recombinant human activated factor VII and canine fresh frozen plasma in Beagles with hereditary coagulation factor VII deficiency. Journal of Veterinary Internal Medicine. 2006; 20:766. (Abstract).

\section{Appendix A. Supplementary material}

Supplementary data associated with this article can be found, in the online version, at doi: 10.1016/j.tvjl.2010.11.010. 


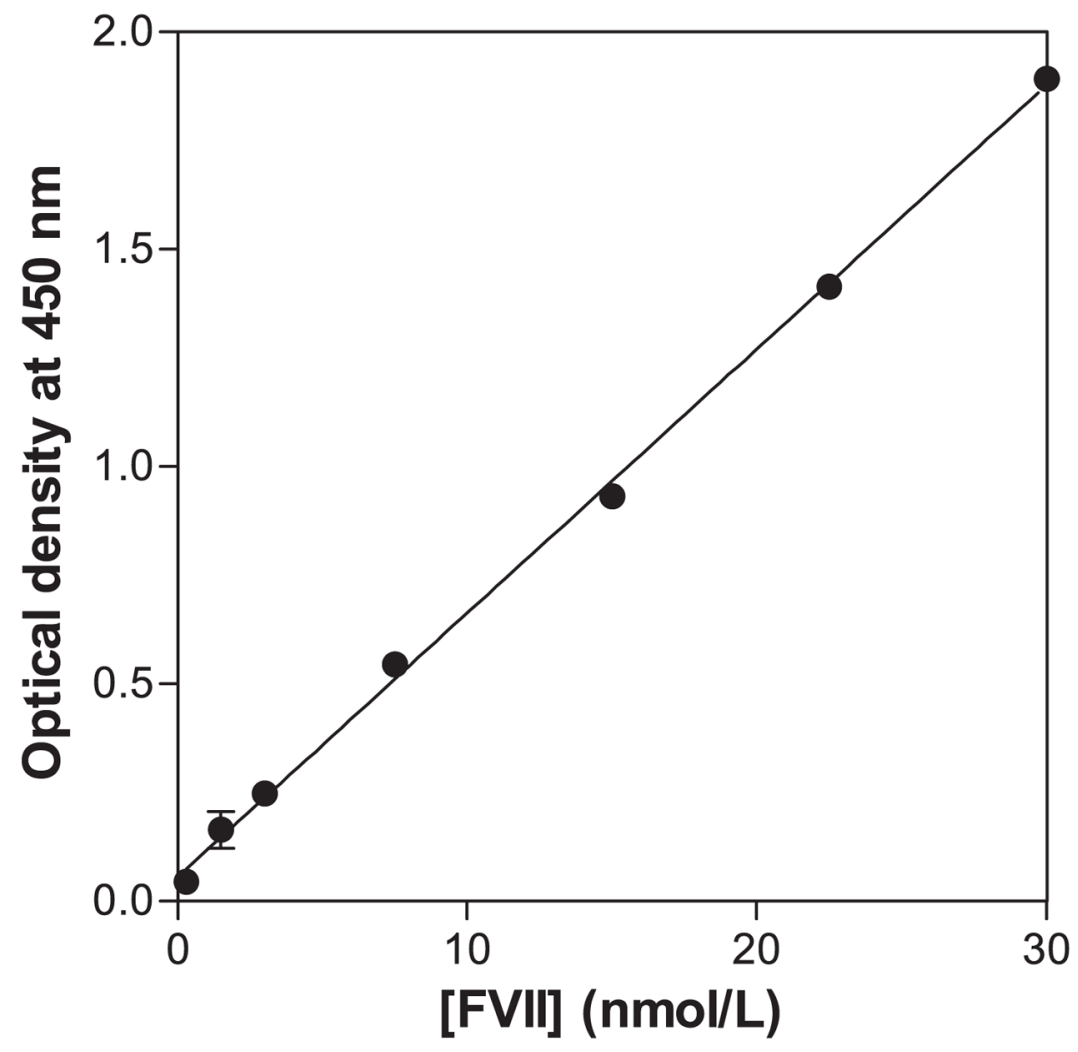

Fig. 1.

Standard curve for the canine specific FVII:Ag method. Optical density readings at $450 \mathrm{~nm}$ obtained from duplicate measurements of samples with $0.3-30 \mathrm{nmol} / \mathrm{L}$ recombinant canine FVII were fitted to a second order curve and used to calculate FVII:Ag in samples. A typical curve is shown $\left(r^{2}=0.998\right)$. 


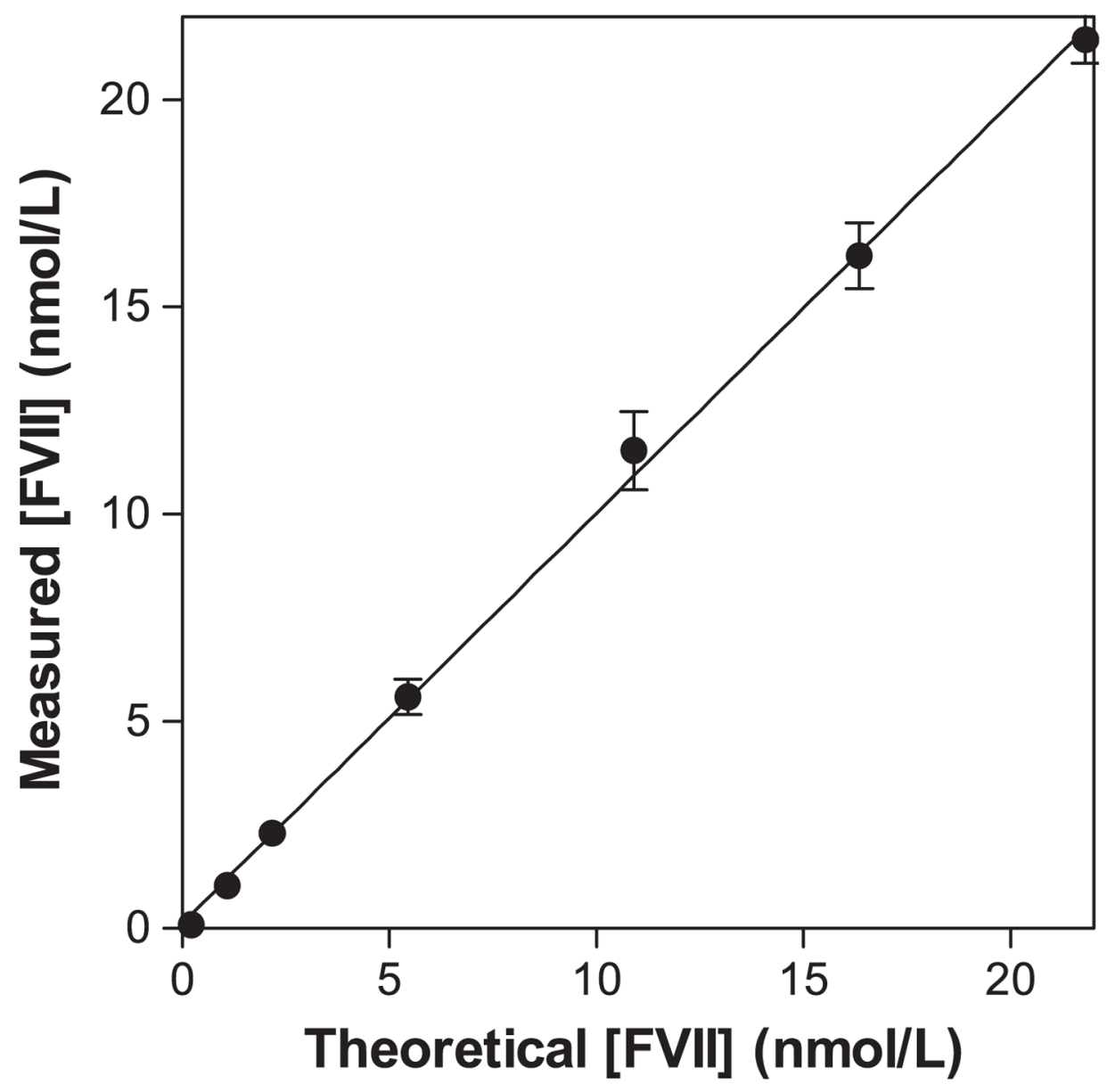

Fig. 2.

Dilution linearity for the canine specific FVII:Ag method. Dilution linearity met criteria $\left(r^{2}\right.$ $>0.99$, slope and intercept not statistically different from 1 and 0 , respectively) as assessed by linear regression of data obtained in three runs with duplicate measurements of serially diluted normal plasma; $r^{2}=0.995$, slope $\left(\mathrm{CI}_{95}\right)=[0.956 ; 1.024]$, intercept $\left(\mathrm{CI}_{95}\right)=[-0.275$; $0.518]$. 

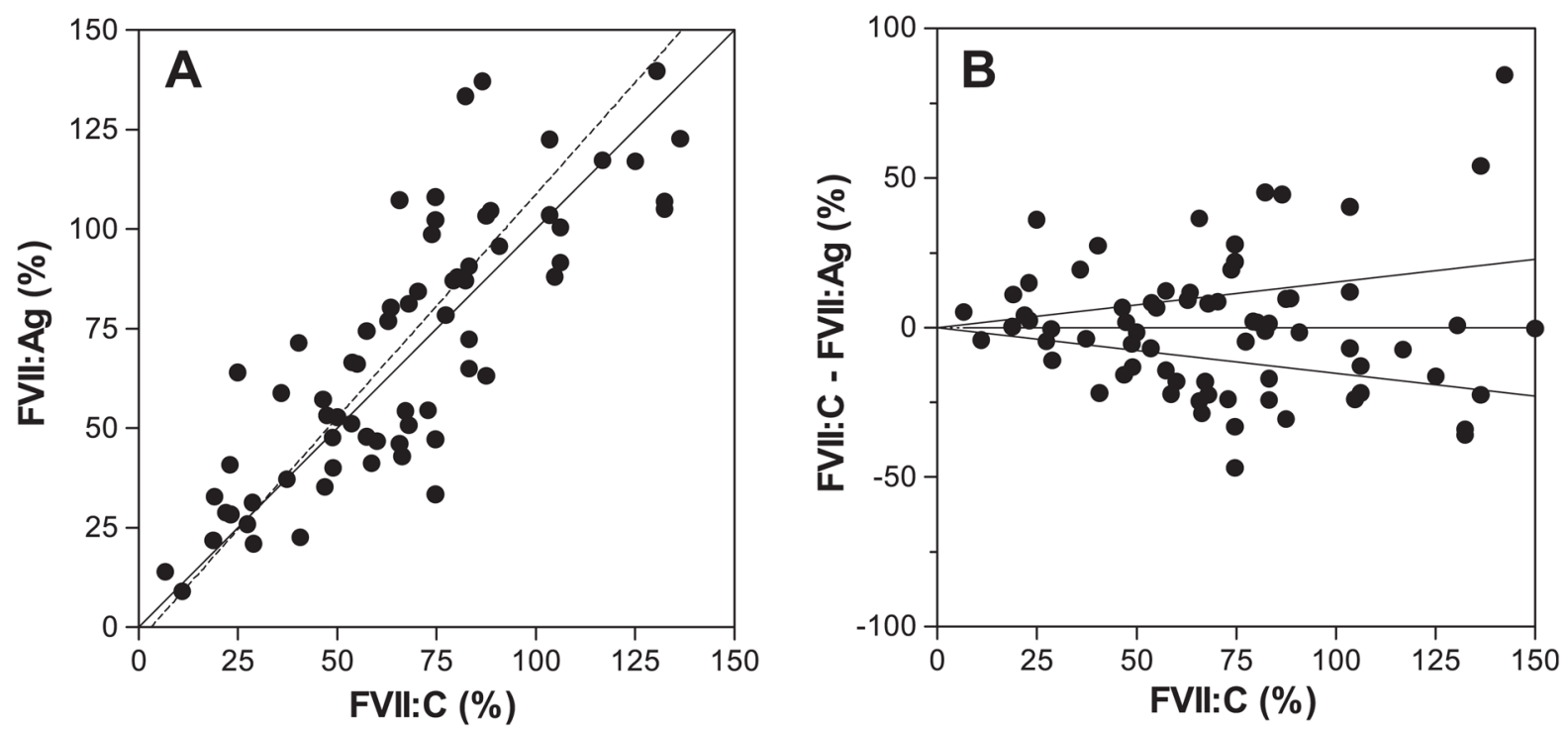

Fig. 3.

Comparison of FVII:C and FVII:Ag methods to measure FVII in plasma. (A) Statistically significant correlation between FVII:C and FVII:Ag measurements was observed with clinical samples from dogs with clinical babesiosis $\left(r=0.843, \mathrm{CI}_{95}\right.$ : [0.758; 0.899]; $P<$ $0.0001 ; n=71$ ). When compared to total agreement (solid line), Passing-Bablock linear regression (dotted line) did not identify disagreement between assays $(P=0.5)$ and no deviation from linearity was identified $(P>0.10)$. (B) Bland-Altman plot with differences between FVII:C and FVII:Ag vs. FVII:C $(n=71)$. Forty-three differences $(61 \%)$ in total were outside (19 above, 27\%; 24 below, 34\%) bounds (lines) for combined inherent imprecision of the assays. 

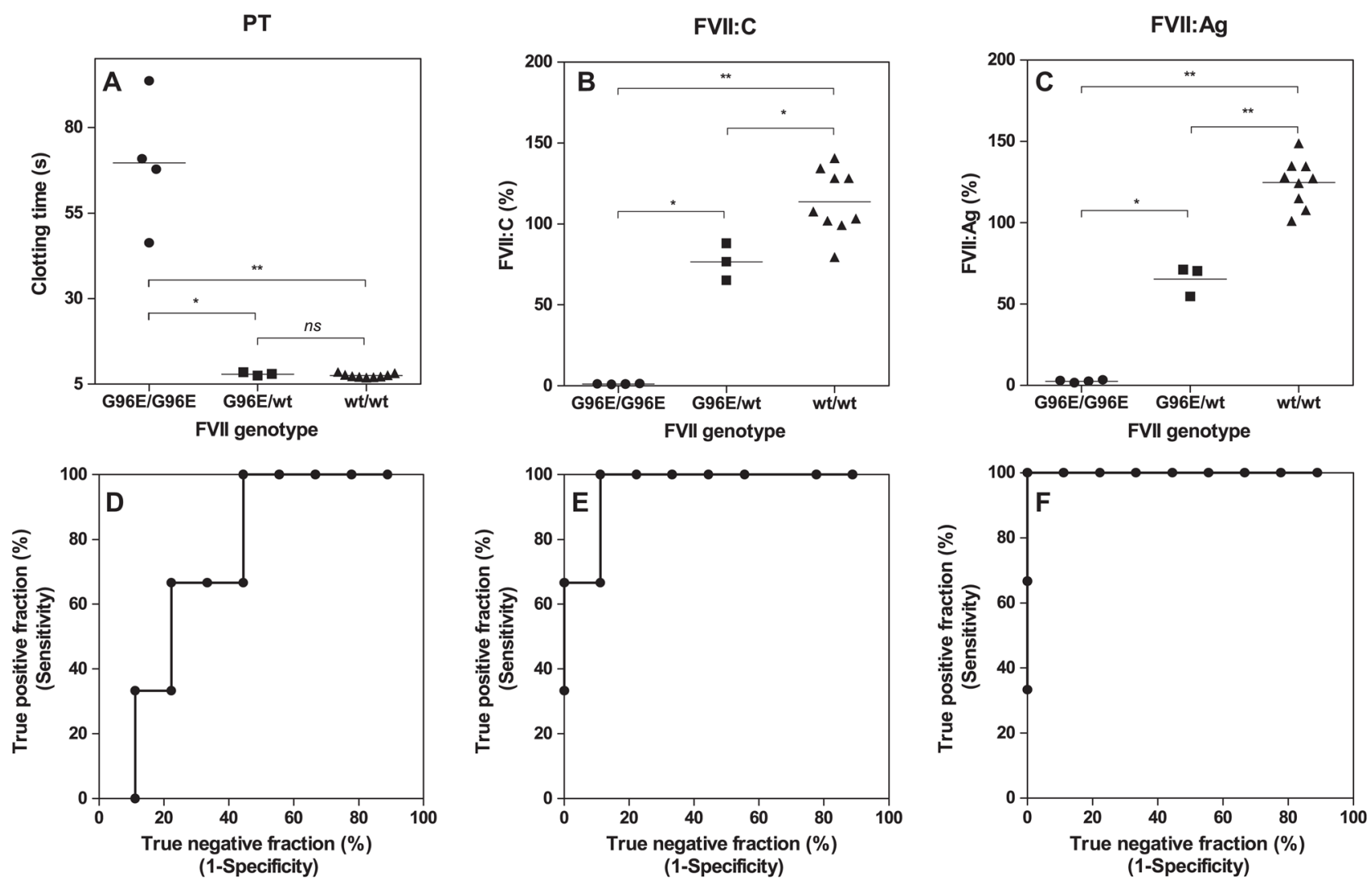

Fig. 4.

Diagnostic performance of three different plasma based assays to detect hereditary FVII deficiency. Upper panel: Plasma FVII measured by (A) prothrombin time (PT), (B) FVII activity (FVII:C) and (C) FVII antigen (FVII:Ag) in plasma from dogs with three different FVII genotypes. ${ }^{*} P<0.05,{ }^{* *} P<0.005$. Lower panel: Receiver operator characteristic (ROC) curve for distinction between normal dogs and FVII deficient heterozygotes, i.e. disease gene carriers. To demonstrate diagnostic capability of an assay, the area under the ROC curve $\left(\mathrm{AUC}_{\mathrm{ROC}}\right)$ must be $>0.5$. Lower $95 \%$ confidence interval limits were (D) 0.453 for PT, (E) 0.857 for FVII:C and (F) 1.0 for FVII:Ag. In comparison, $\mathrm{AUC}_{\mathrm{ROC}}$ was 1.0 for distinction between normal dogs and FVII deficient homozygotes, as well as for distinction between by FVII deficient hetero- and homozygotes for all three assays. Data represent means of duplicate measurements. 


\section{Table 1}

Analytical imprecision within and between assays.

\begin{tabular}{lrllrr}
\hline \multirow{2}{*}{ Specimen } & \multicolumn{2}{l}{ Intra-assay $\boldsymbol{n}=\mathbf{1 0}$ (singlets) } & & \multicolumn{2}{l}{ Inter-assay $\boldsymbol{n}=\mathbf{5}$ (duplicates) } \\
\cline { 2 - 3 } \cline { 5 - 6 } & FVII:Ag (nmol/L) & $\mathbf{C V \%} \boldsymbol{a}$ & FVII:Ag (nmol/L) & $\mathbf{C V \%} \boldsymbol{a}$ \\
\hline Low & 2.0 & 6.1 & & 2.0 & 10.0 \\
Medium & 11.4 & 3.2 & & 12.3 & 7.9 \\
High & 23.8 & 2.6 & & 23.0 & 5.1 \\
\hline
\end{tabular}

FVII:Ag represents mean values.

${ }^{a} \mathrm{CV}$, coefficient of variation. 


\section{Table 2}

Recovery of low, medium and high canine FVII:Ag in different matrixes (\%).

\begin{tabular}{llll}
\hline & Low & Medium & High \\
\hline Canine FVII deficient plasma & 97 & 106 & 106 \\
Human normal and FVII deficient plasma & 91 & 105 & 105 \\
Buffer & 96 & 102 & 100 \\
\hline
\end{tabular}

Theoretical FVII:Ag after spiking with 3 (low), 15 (medium) and 27 (high) nM recombinant canine FVII defined as 100\%. Data are baseline corrected and reported as mean of individual runs $(n=3)$ with duplicate measurements in each run. 


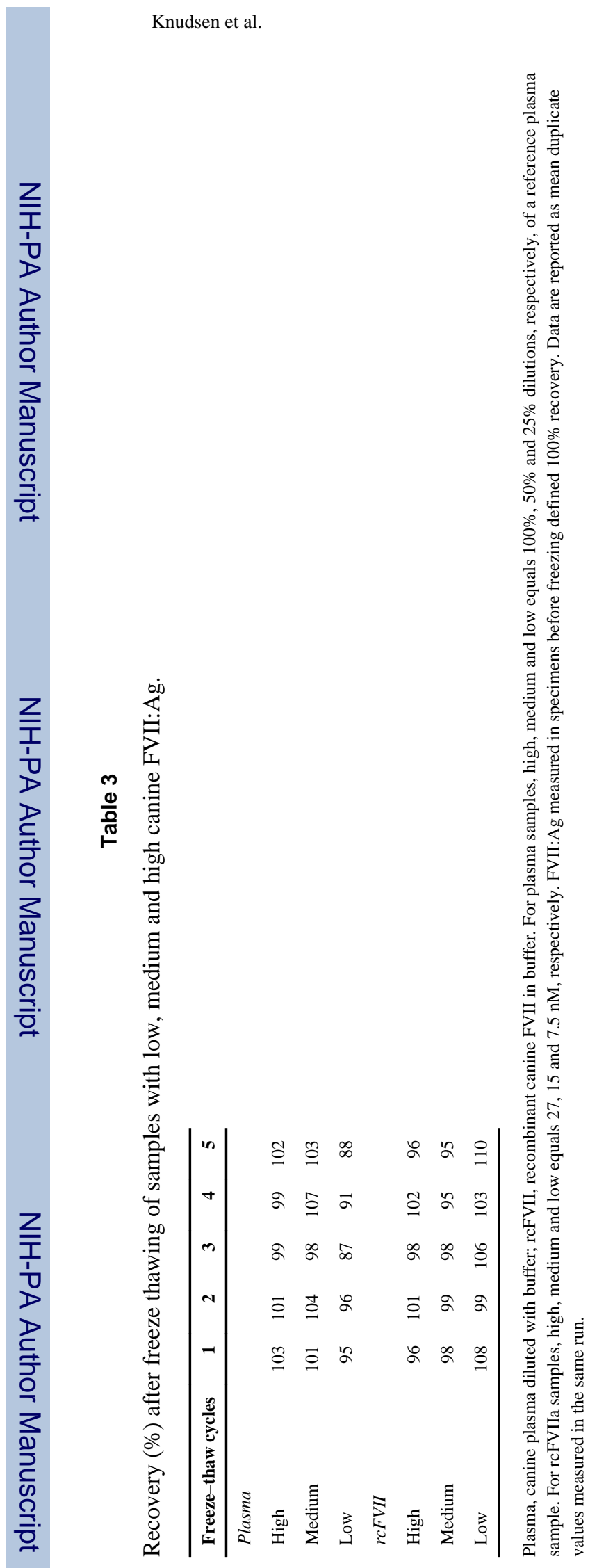

Vet J. Author manuscript; available in PMC 2014 March 09. 


\section{Table 4}

Mean and range of prothrombin time, FVII activity and antigen in dogs with homozygous (G96E/G96E), heterozygous (wt/G96E) and without (wt/wt) FVII deficiency.

\begin{tabular}{llll}
\hline & G96E/G96E $(\boldsymbol{n}=\mathbf{4})$ & $\mathbf{w t} / \mathbf{G 9 6 E}(\boldsymbol{n}=\mathbf{3})$ & $\mathbf{w t} / \mathbf{w t}(\boldsymbol{n}=\mathbf{9})$ \\
\hline PT (s) & $70(46-94)$ & $8.0(7.5-8.5)$ & $7.5(7.0-8.6)$ \\
FVII:C (\%) & $1.1(0.9-1.3)$ & $76(65-88)$ & $114(80-141)$ \\
FVII:Ag (\%) & $2.6(1.6-3.4)$ & $65(55-71)$ & $125(101-149)$ \\
\hline
\end{tabular}

Dogs were grouped according to FVII genotype. Data are mean (range) of duplicate measurements. Prothrombin time is reported as clotting time in seconds. FVII activity (FVII:C) and antigen (FVII:Ag) are given relative to pooled normal canine plasma defining $100 \%$. 